\title{
Aggregation-induced emission spectral shift as a measure of local concentration of a pH-activatable rhodamine-based smart probe
}

\author{
Zoran Arsov $^{1,2}$ *, Iztok Urbančič ${ }^{1}$, Janez Štrancar ${ }^{1,2}$ \\ ${ }^{1}$ Laboratory of Biophysics, Department of Condensed Matter Physics, Jozef Stefan Institute, Ljubljana, \\ Slovenia \\ ${ }^{2}$ Center of Excellence NAMASTE, Ljubljana, Slovenia \\ *Corresponding author: zoran.arsov@ijs.si
}

\section{$\underline{\text { Abstract }}$}

Generating activatable probes that report about molecular vicinity through contact-based mechanisms such as aggregation can be very useful. Specifically, such probes change a particular spectral property only at the intended biologically relevant target. Xanthene derivatives, for example rhodamines, are able to form aggregates. It is typical to examine aggregation by absorption spectroscopy but for microscopy applications utilizing fluorescent probes it is very important to perform characterization by measuring fluorescence spectra. First we show that excitation spectra of aqueous solutions of rhodamine $6 \mathrm{G}$ can be very informative about the aggregation features. Next we establish the dependence of the fluorescence emission spectral maximum shift on the dimer concentration. The obtained information helped us confirm the possibility of aggregation of a recently designed and synthesized rhodamine 6G-based pH-activatable fluorescent probe and to study its $\mathrm{pH}$ and concentration dependence. The size of the aggregationinduced emission spectral shift at specific position on the sample can be measured by fluorescence microspectroscopy, which at particular $\mathrm{pH}$ allows estimation of the local concentration of the observed probe at microscopic level. Therefore, we show that besides aggregation-caused quenching and aggregation-induced emission also aggregationinduced emission spectral shift can be a useful photophysical phenomenon.

Keywords: rhodamine; activatable probes; aggregation; dimerization; aggregation-induced emission spectral shift 


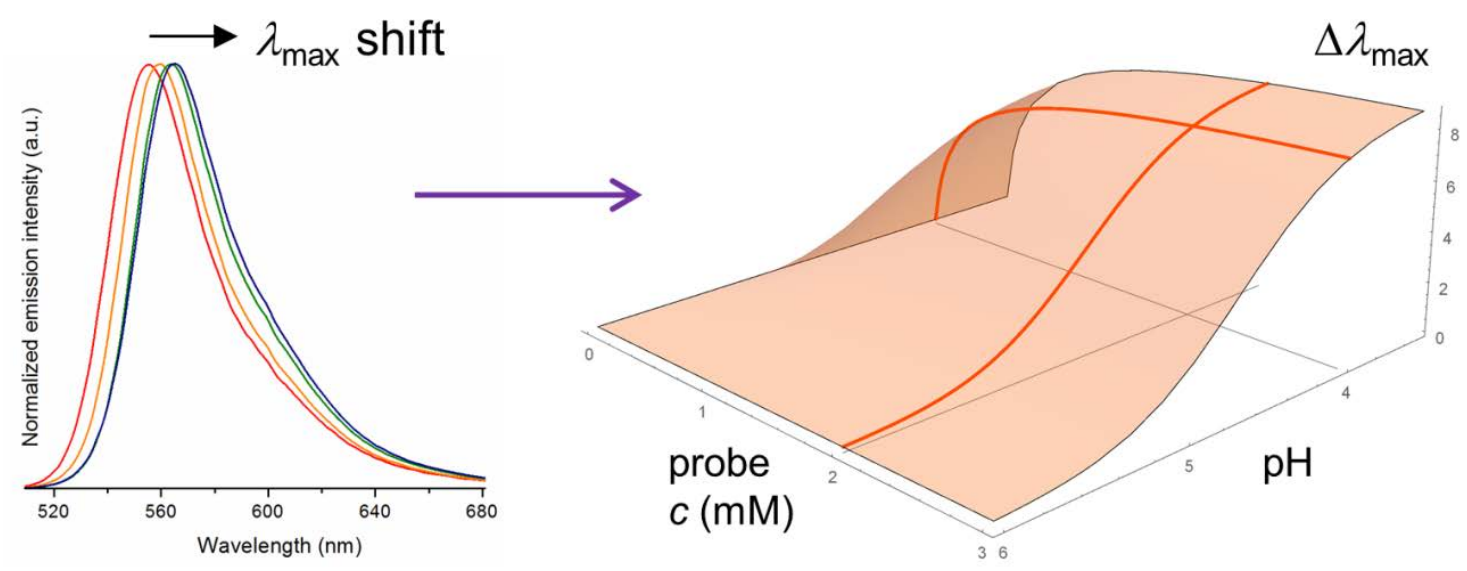

\section{INTRODUCTION}

A desirable property of fluorescent probes is that they can be activatable, i.e. that they become fluorescent [1] or change a particular spectral property only at the intended target. With such smart probes the background signal is minimized and thus target to background ratios can be improved. There are a number of mechanisms for generating activatable probes that probe molecular vicinity or molecular confinement, but all require some kind of efficient intensity reduction or spectral change. Widely used is homo- or hetero-Förster resonance energy transfer (FRET). As an alternative, contact-based mechanisms are also possible [2-4]. For example, in the case of xanthene derivatives, such as rhodamines, aggregate formation can lead to aggregation-caused quenching (ACQ) [5], spectral shifts [6], and aggregation-induced emission (AIE) [7].

According to the exciton theory [8] the monomer excited-state levels split in two upon dimerization. This splitting depends on the arrangement, i.e. on the angle and the distance, of the transition dipoles of the molecules in the dimer. Consequently, possible transitions from the ground state to either of the excited states depend on the geometry of the dimer. For parallel or sandwich type dimers, denoted as $\mathrm{H}$-dimers, the transition to the lower energy excited state is forbidden. For head-to-tail or in-line dimers, denoted as Jdimers, the transition to the higher energy excited state is forbidden. In dimers having 
intermediate geometries, both transitions are partially allowed. Besides probe concentration and structure, the level of aggregation and the attained geometry can depend on several other conditions that can modify intermolecular interaction such as temperature, polarity of the solvent, presence of particular ionic species or macromolecules and ionic strength $[5,9,10]$. For example, by changing polarity of the solvent with ethylene glycol [11], the energy levels and geometries can be affected [12].

The most typical approach in studying a fluorescent dye or probe aggregation is to study absorbance spectra [5]. Historically such studies were propelled by the use of rhodamine dyes as an active medium in lasers. Knowledge of its association properties was important because greater self-absorption and fluorescence quenching weakened the laser output power [13]. The shape of absorbance spectra also reveals the types of dimers formed. Especially formation of $\mathrm{H}$-dimers can nicely be discerned, since a decrease in the monomer absorption and an appearance of a new blue-shifted shoulder/peak at lower wavelengths are observed [5]. On the other hand, J-dimers are more strongly overlapped by the monomer peak so it is more difficult to discern their presence $[10,14]$ This could probably be the reason why in aqueous solutions aggregation effects were attributed mainly to the formation of non-fluorescent $\mathrm{H}$-dimers.

So it was often assumed that rhodamines form almost exclusively non-fluorescent $\mathrm{H}$-dimers in aqueous solutions [5,15]. But then different experiments showed that in aqueous solutions rhodamines show intermediate and deviated geometries from ideal $\mathrm{H}$ dimers and J-dimers [16,17], therefore spectroscopic characteristics of both $\mathrm{H}$ - and J-type aggregates should be detected. The reason for this is that in the case of rhodamine dyes, the aromatic xanthene ring systems are not symmetrical. As a consequence, different dimers may be formed and the optical selection rules are relaxed. Both transitions (to the higher as well as to the lower dimer excited state) can then be observed [18].

Because of the expected non-fluorescent nature of $\mathrm{H}$-dimers and quenching of the monomer emission it is expected that the presence of J-dimers would be more easily discerned in fluorescence spectra. The number of studies in this direction is much more limited. Especially limited are the studies of dependence of excitation spectra on dye concentration. A few excitation studies exist on adsorbed rhodamine systems $[10,14,19,20]$, however they are scarce on aqueous solutions [18]. Also emission spectra reflect very interesting concentration-dependent effects. But similarly, the emission studies are also rare $[17,21]$, although they can offer insight into the nature of vicinity effects. Beside obtaining complementary information of the mechanisms of aggregation, fluorescence spectroscopic information are particularly valuable since applications of 
fluorescent probes for imaging rely on measuring and characterizing fluorescence emission. Although absorption studies are very informative for characterization of the molecular behavior in solution, the translation of such approach to microscopy of complex environments, such as living cells, is rather limited due to the lack of instrumentation to measure spatially-resolved absorption with adequate resolution. On the other hand, microscopes offering spectral characterization of emitted fluorescence are becoming widespread [22], including super-resolved approaches [23]. To fill the above mentioned gap, we present both fluorescence excitation and emission spectra for aqueous solutions of rhodamine 6G (R6G) and an R6G-based fluorescent imaging probe.

Because of the stated relevance of imaging applications, it is especially useful to examine the concentration dependence of the emission spectra. Several studies showed that the presence of R6G aggregates (dimers) can lead to a red-shift in the emission spectrum in different environments $[6,10,19]$. For example, even in the case of concentrated aqueous solutions of R6G, a typical example of what was thought to lead to the formation of $\mathrm{H}$-dimers with blue-shifted absorbance spectra, the red-shift in the emission spectra can be observed [21]. It is valuable to relate the dimer concentration with the magnitude of red-shift.

A similar shift has been recently observed in our study of internalization of a smart fluorescent probe by antigen-presenting dendritic cells (DCs) [24]. The antigen presentation is initiated by receptor internalization to endosomes and lysosomes. The interior of these organelles is acidic compared to mainly neutral $\mathrm{pH}$ of cytosol. Therefore, to study the internalization mechanism, an R6G-based fluorescent probe, abbreviated in this paper as RDC, that combines a DC-characteristic receptor ligand and a $\mathrm{pH}$-activatable dye has been designed and synthesized. Beside activation in a low-pH environment due to a structural change in the probe, it was very interesting to observe an aggregation-induced spectral shift that pointed to the accumulation of RDC in low-pH cellular structures.

The aim of this work was to provide more insight into the effect of aggregation of rhodamine-based probes in aqueous solutions on the shape of fluorescence excitation and emission spectra and in this way supplement existing knowledge obtained by absorbance spectroscopy. Since in imaging applications fluorescence emission is followed, a particular emphasis was given to the understanding of dependence of the extent of emission spectral red-shift on concentration (and $\mathrm{pH}$ in the case of RDC). R6G was first characterized. Although R6G dimers in aqueous solutions are expected to form mostly parallel geometries and subsequently should be less fluorescently active, a clear effect of aggregation is observed in both excitation and emission spectra. The observed effects 
were compared to the measurements of RDC, which showed similar behavior. It was possible to relate the amount of shift of the fluorescence emission spectral maximum to the mole fraction of dimers. Moreover, the magnitude of the shift can also be related to the $\mathrm{pH}$ of the environment. The obtained data can be used as a reference to assess the local concentration of RDC probe at specific position on the sample during imaging without the problem of local intensity normalization.

\section{MATERIALS AND METHODS}

\section{$\underline{2.1 \text { Materials }}$}

All the chemicals used were obtained from commercial sources and used without further purification. Solvents used as reaction media were absolute grade and used as received. R6G was purchased from Sigma. The $\mathrm{pH}$-activatable probe RDC was synthesized as described previously [24].

\subsection{Measurements of fluorescence excitation and emission spectra}

Fluorescence spectra were measured with a Tecan Infinite M1000 microplate reader close to room temperature at $28{ }^{\circ} \mathrm{C} \pm 1{ }^{\circ} \mathrm{C}$. A 96-well plate was used in the fluorescence intensity top mode with a filling volume of $50 \mu$ l. The excitation from 400 to $580 \mathrm{~nm}$ was recorded (10-nm band pass) at the emission wavelength $610 \mathrm{~nm}(10-\mathrm{nm}$ band pass) in 2 or $4 \mathrm{~nm}$ steps. The emission spectra from 480 to $700 \mathrm{~nm}$ were recorded (10-nm band pass) with the excitation wavelength $450 \mathrm{~nm}$ (10-nm band pass) in 2 or $4 \mathrm{~nm}$ steps. The wavelength accuracy, as reported in the technical specifications, is $\pm 1 \mathrm{~nm}$.

The quantity we mainly focus on in this paper is the fluorescence emission spectral maximum $\lambda_{\max }$. It is obtained by fitting a central part of the emission spectra with a fourth order polynomial and determining its maximum position. Such procedure reduces the impact of experimental and instrumental noise on the out-coming results. A control experiment was conducted to evaluate the precision of this approach. Emission spectra from R6G solutions were recorded on the microplate reader several times in 10-min 
intervals at stabilized temperature (at $31 \pm 0.3^{\circ} \mathrm{C}$ ). Under these conditions, experimentally determined variability in $\lambda_{\max }$ was less than $\pm 0.1 \mathrm{~nm}$.

The top mode measurements enabled inspection at higher probe concentrations, since the inner filter effects can be avoided or reduced. For moderate or high dye concentrations self-absorption (also called reabsorption) of the emitted light may affect the recorded spectral shape [25]. To further reduce a possibility of the self-absorption, the Zposition of the fluorescence top optics was manually set to the highest value. To check for self-absorption effects a control measurement was done in which the Z-position was systematically decreased. Even though the effective size of the excitation volume increased by reducing the Z-position the shape of the spectra did not change significantly. In the case of substantial re-absorption effects the shape would be dependent on the distance that fluorescence emission light is traveling inside the sample. For both excitation [26] and emission $[6,21,26,27]$ previous experiments showed that it is possible to exclude or limit these effects.

On the other hand, due to its limited sensitivity, the microplate reader is not well suited for measurements at lower concentrations. Consequently, fluorescence spectra for low concentrations of RDC were measured using a PerkinElmer LS 55 fluorescence spectrometer at room temperature.

A possible influence of temperature on the measurements with the microplate reader was tested. The temperature dependence studies of fluorescence spectral properties of R6G in aqueous solutions are rare and mostly focused on intensity. We therefore conducted measurements from $23{ }^{\circ} \mathrm{C}$ to $38{ }^{\circ} \mathrm{C}$, approximately in the range provided by the microplate reader. Results are presented in Table 1 and are in line with previously published observations for intensity [28]. It can be seen that the degree and direction of variation depends on the dye concentration for both intensity and $\lambda_{\max }$, which is a consequence of aggregation [28,29]. Both quantities changed steadily and approximately linearly in the studied temperature interval so only comparison of the starting and the ending values are shown in Table 1. Based on these results the temperature variation during measurements $\left( \pm 1{ }^{\circ} \mathrm{C}\right)$ might cause an additional $\lambda_{\max }$ variability of up to $\pm 0.1 \mathrm{~nm}$,

Citrate/phosphate buffer, which covers a very broad range of $\mathrm{pH}$ values, was used in experiments. The value of $\mathrm{pH}$ is varied by modification of the citrate/phosphate ratio. The RDC probe was non-fluorescent in $\mathrm{pH}$ neutral solution. As the $\mathrm{pH}$ decreased, the fluorescence intensity increased greatly and the buffer solution turned pink. 
Table 1. Temperature dependence of fluorescence emission spectrum intensity $I$ and $\lambda_{\max }$ of R6G solutions in citrate/phosphate buffer at $\mathrm{pH}=3.5$

\begin{tabular}{|l|l|l|l|l|}
\hline & $c=0.01 \mathrm{mM}$ & $c=0.1 \mathrm{mM}$ & $c=0.4 \mathrm{mM}$ & $c=1 \mathrm{mM}$ \\
\hline$I^{38} / I^{231}$ & 0.88 & 1.01 & 1.12 & 1.11 \\
\hline$\lambda_{\max }^{38}-\lambda_{\max }{ }^{23}(\mathrm{~nm})$ & 0.5 & 0.8 & 1.4 & 1.4 \\
\hline
\end{tabular}

${ }^{1}$ The superscripts 23 and 38 denote the measurement temperatures, $23^{\circ} \mathrm{C}$ and $38^{\circ} \mathrm{C}$, respectively.

\subsection{Data analysis}

At higher concentration of rhodamine-based probes aggregation can occur in aqueous solutions. If concentrations are low enough to avoid trimer and higher-order aggregate formation, then we have an equilibrium between monomers and dimers

$$
M+M \rightleftharpoons D \text {. }
$$

The association (or in this case dimerization) constant $K_{\mathrm{D}}$ is defined as

$$
K_{\mathrm{D}}=c_{\mathrm{D}} / c_{\mathrm{M}}^{2} \text {, }
$$

where $c_{M}$ is monomer concentration, $c_{D}$ dimer concetration and $c=c_{M}+2 c_{D}$ the total concentration of a probe. The mole fraction of dimers can be derived as (see for example [6])

$$
x_{\mathrm{D}}\left[c, K_{\mathrm{D}}\right]=\frac{2 c_{D}}{c}=\frac{4 K_{\mathrm{D}} c+1-\sqrt{8 K_{\mathrm{D}} c+1}}{4 K_{\mathrm{D}} c} .
$$

For analysis of a $\mathrm{pH}$ dependence the sigmoid curve in the form of the logistic function can be used

$$
S(\mathrm{pH})=\frac{1}{1+e^{k\left(\mathrm{p} K_{\mathrm{a}}-\mathrm{pH}\right)}},
$$

where $\mathrm{p} K_{\mathrm{a}}$ is the $\mathrm{pH}$ value of the sigmoid's midpoint, and $k$ is the steepness of the curve. The curve's maximum value was treated separately as part of other quantities contributing to the scaling factors of particular fitting or modeling functions. 


\section{RESULTS AND DISCUSSION}

\subsection{Effect of aggregation on fluorescence excitation and emission spectra of R6G}

Typically, the aggregation of dyes is studied by absorbance spectroscopy. Much less studies were conducted by examining fluorescence excitation and emission spectra. Therefore, we studied dependence of the shapes of these spectra on the dye/probe concentration.

We first examined R6G, since this dye is well studied and because it was used in the synthesis of our smart probe. The aggregation effect can be nicely discerned from the excitation spectra (Fig. 1A). Here the spectrum for $0.01 \mathrm{mM}$ concentration (red curve in Fig. 1A) will be regarded as a reference representing predominantly spectroscopic response by monomers. There are two main features observed in the excitation spectra. Firstly, a blue-shifted shoulder with respect to the main monomer peak appears and moves to higher wavelengths with the concentration. Secondly, a red-shifted shoulder emerges and becomes more pronounced with the concentration. Both these features have been observed before $[10,14,18,19]$.
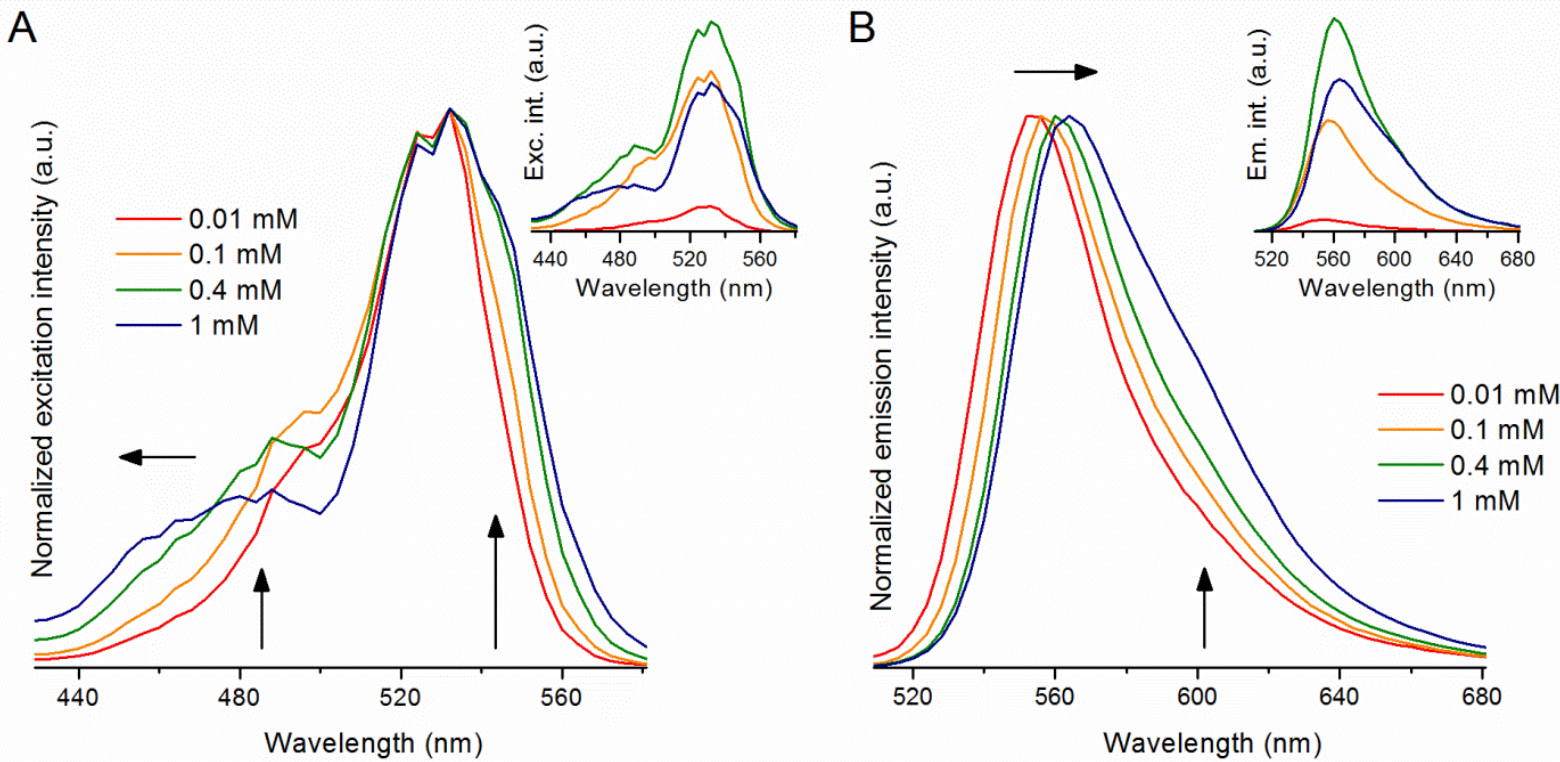

Fig. 1. Dependence of the shape of fluorescence $(A)$ excitation and $(B)$ emission spectra on the concentration of R6G. The spectra in the main part of the figure are normalized to the maximum intensity. Insets show non-normalized spectra. 
Although it was assumed before that non-fluorescent $\mathrm{H}$-dimers prevail in polar solvents whereas J-dimers are characteristic for weakly polar solvents or in the adsorbed state [15], the observed red-shifted shoulder in the excitation spectra of aqueous solutions of R6G reveals effect that is usually assigned to the presence of fluorescent J-dimers [26]. Moreover, the possibility to record the excitation spectra supports by itself the fluorescent character of aggregates, since non-fluorescent species should not show any type of excitation spectrum [26]. Therefore, such observations enable better understanding of intermolecular interactions in rhodamine-based probes.

Nevertheless, a significant part of the formed dimers are non-fluorescent $\mathrm{H}$-dimers or have $\mathrm{H}$-dimer-like properties. Consequently, the presence of the blue-shifted shoulder is unexpected, because it means that emission is detected with excitation at shorter wavelengths. This behavior was explained by depopulation of the excited higher energy levels of dimers (H-dimers) through a non-radiative deactivation process (energy transfer and/or non-radiative internal conversion process) to the fluorescent lower energy levels of dimers (J-dimers) $[18,20]$.

Because of the non-radiative transition of $\mathrm{H}$-dimers from excited to ground state no blue-shifted component is observed in the emission spectra (Fig. 1B). The main effect of formation of aggregates in aqueous solutions is the red-shift, as observed also before [21]. Else the shape of the spectrum does not change significantly except for the highest concentration. The reason for this is probably that the components comprising the spectra and corresponding to the monomer and dimer emission have similar shapes, only that the dimer component is red-shifted [6]. The overall red-shift is a consequence of increased proportion of the dimer component with higher R6G concentration. Also the change of excitation spectra as a function of dye concentration indicates that the formation of fluorescent dye aggregates is responsible for the emission changes [14].

Both excitation and emission spectra reveal quenching at higher dye concentrations (see Fig. 1, insets) as reported before [27,30]. Aggregation-caused quenching decreases the emission quantum yield of R6G by a combination of transfer of excitation energy between monomers and aggregates [18,31,32] and absorption of radiation by nonfluorescent dimers $[5,18]$, which come to the ground state in a radiationless process.

In Fig. 2 the concentration dependence of the shift of the fluorescence emission spectral maximum $\Delta \lambda_{\max }$ is presented. $\Delta \lambda_{\max }$ is calculated with respect to the reference $\lambda_{\max }$ at $\mathrm{c}=0.01 \mathrm{mM}$, so the first measurement point in Fig. 2 is shown without an error bar. The shape of the emission spectrum can be assumed to be a linear superposition of the components corresponding to the monomer emission spectrum and the dimer spectrum as 
discussed above. Dimerization is presumed to be the dominant form of aggregation in the studied concentration range [33], although at $1 \mathrm{mM}$ some trimers can already be present [34]. So it can be reasonably expected that the spectral shift will depend on the mole fraction of dimers. The dependence of $\Delta \lambda_{\max }$ is assumed to be proportional to the mole fraction of dimers

$$
\Delta \lambda_{\max }(c)=A x_{\mathrm{D}}\left[c, K_{\mathrm{D}}\right]
$$

where $x_{D}$ is taken into account according to Eq. (3). The scaling factor $A$ reflects the magnitude of the spectral maximum shift. A close agreement between experimental data and the fit (solid line in Fig. 2) supports the above assumption. Moreover, measurement uncertainties are in the range of a few tenths of $\mathrm{nm}$, so much smaller than the observed $\Delta \lambda_{\max }$ changes. At the total concentrations where dimers start to form the aggregation effect is the greatest, hence the steep rise in the first part of the figure. With extensive formation of dimers the pool of monomers decreases, which leads to a saturated-like shape in the second part.

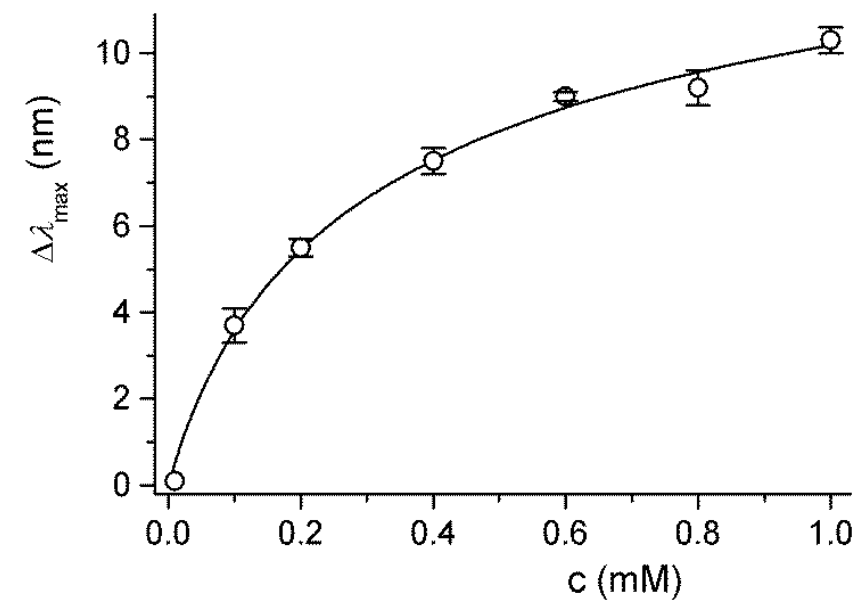

Fig. 2. Dependence of the fluorescence emission spectral maximum shift $\Delta \lambda_{\max }$ on the concentration of R6G. Each point represents an average value from two different experiments. Error bars represent estimate of the uncertainty in the $\Delta \lambda_{\max }$ shift. The line represents a fitting curve according to Eq. (5).

The value of the dimerization constant $K_{D}=1.9 \pm 0.2 \mathrm{mM}^{-1}$ obtained from the fit is in accordance with previously published values for R6G in aqueous solutions at room temperature. For example the values $1.7 \mathrm{mM}^{-1}$ [5], $2.6 \mathrm{mM}^{-1}$ [34], $1.7 \mathrm{mM}^{-1}$ [35], and 1.8 $\mathrm{mM}^{-1}$ [36] were obtained, although a higher value of $6.2 \mathrm{mM}^{-1}$ was also reported [13]. It has 
to be noted that the relation between $\Delta \lambda_{\max }$ and $c$ postulated in Eq. (5) is only empirical, since we do not know exactly how the concentration of dimers affects the emission spectrum shape.

\subsection{Effect of aggregation on fluorescence excitation and emission spectra of RDC}

Similar experiments were repeated for the pH-sensitive synthesized R6G-based RDC probe to compare with the obtained results for R6G. The process of aggregation is revealed from effects similar to R6G in both types of fluorescence spectra (Fig. 3). A difference can be observed in the excitation spectra (Fig. 3A), where a more pronounced blue-shifted shoulder emerges than for R6G. This discrepancy probably reflects different distribution of the $\mathrm{H}$ - and J-type of dimers or a difference in the non-radiative deactivation process of $\mathrm{H}$-dimers. The behavior of the emission spectra does not show any significant differences (Fig. 3B).
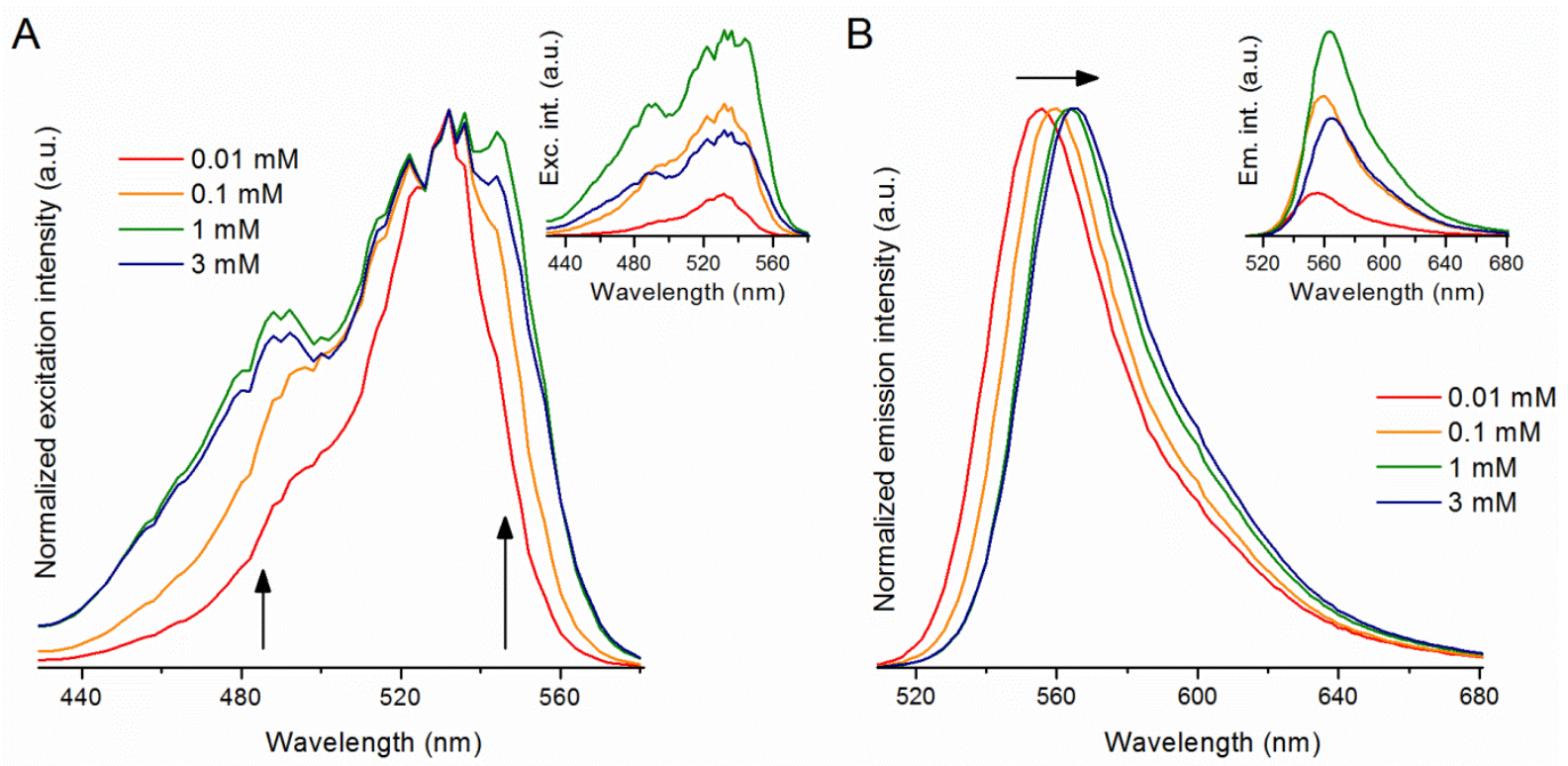

Fig. 3. Dependence of the shape of fluorescence $(A)$ excitation and (B) emission spectra on the concentration of $\mathrm{RDC}$ at $\mathrm{pH}=3.5$. The spectra in the main part of the figure are normalized to the maximum intensity. Insets show non-normalized spectra.

In Fig. 4 the dependence of $\Delta \lambda_{\max }$ on the probe concentration is shown for $\mathrm{pH}=3.5$. $\Delta \lambda_{\max }$ is calculated with respect to the reference $\lambda_{\max }$ at $\mathrm{c}=0.01 \mathrm{mM}$. A similar 
dependence on the dimerization can be observed as in the case of R6G, since a curve according to Eq. (5) nicely fits the data points. The value of the obtained dimerization constant $K_{\mathrm{D}}=3.0 \pm 0.3 \mathrm{mM}^{-1}$ resembles the value for R6G.

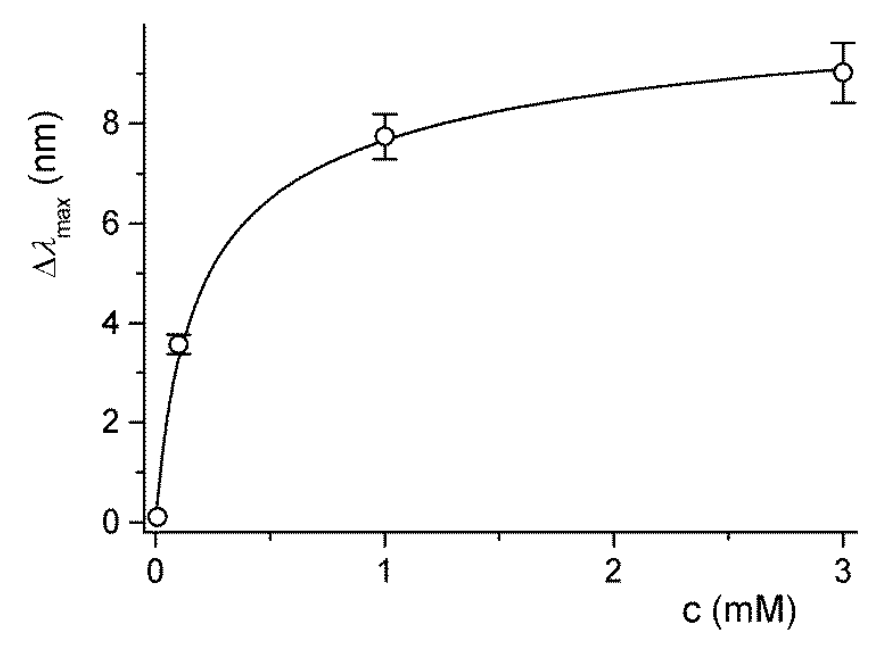

Fig. 4. Dependence of the fluorescence emission spectral maximum shift $\Delta \lambda_{\max }$ on the concentration of $\mathrm{RDC}$ at $\mathrm{pH}=3.5$. Each point represents an average value from two different experiments. Error bars represent estimate of the uncertainty in the $\Delta \lambda_{\max }$ shift. The line represents a fitting curve according to Eq. (5).

\section{$\underline{3.3 \mathrm{Effect} \text { of } \mathrm{pH} \text { on structural and spectral properties of RDC }}$}

The structural and conformational dependence of RDC on pH is shown in Fig. 5. As a starting point for experiments in the previous subsection $\mathrm{pH}=3.5$ was chosen because almost all RDC is in the fluorescent ring-open form (ro-RDC) at this $\mathrm{pH}$. At neutral $\mathrm{pH}$ a completely different behavior is seen due to the spirocyclic form of RDC (sc-RDC). Almost no excitation and consequently also no fluorescence emission is detectable (data not shown). The conversion from sc-RDC to ro-RDC causes rise in the fluorescence emission. 
A

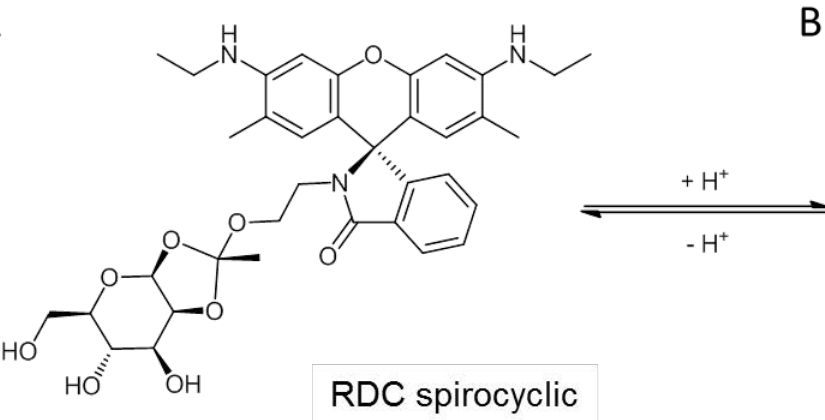

B

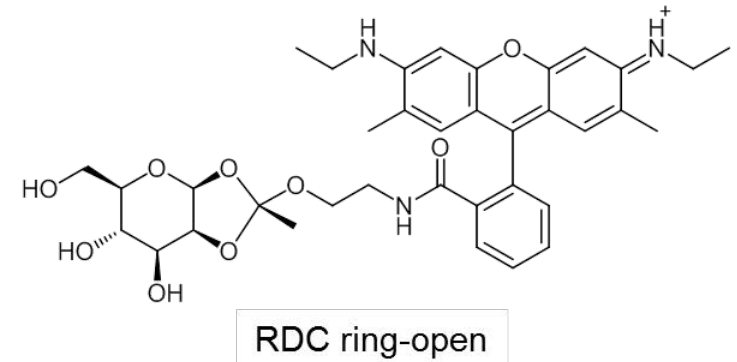

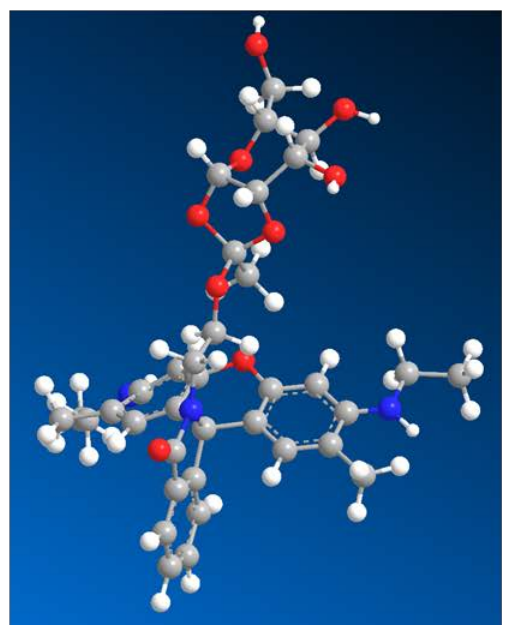

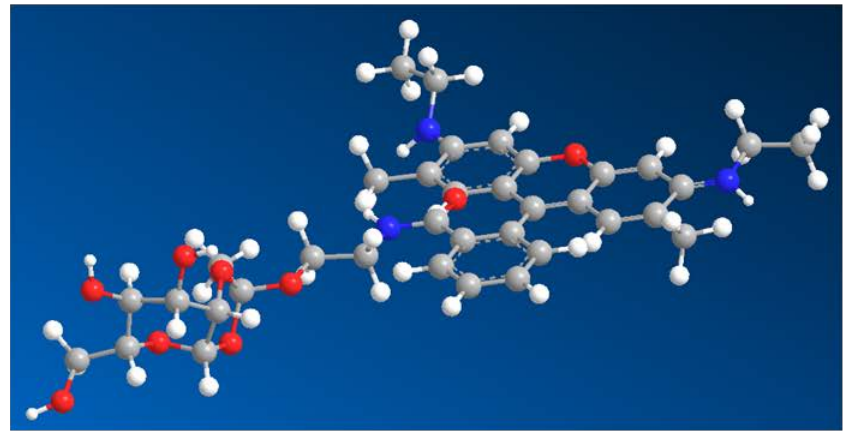

Fig. 5. $\mathrm{pH}$ dependence of RDC structure and conformation: (A) sc-RDC, spirocyclic form, (B) ro-RDC, ring-open form. The structures are shown at the top, 3D representations of the conformation at the bottom.

The $\mathrm{pH}$ dependence of fluorescence emission intensity can be fitted with a sigmoid function from Eq. (4) with $p K_{a}=4.7 \pm 0.1$ (Fig. 6), where the scaling factor represents the maximum emission intensity [37]. The concentration used in this measurement ( $c=0.1$ $\mu \mathrm{M}$ ) was set low on purpose to completely avoid formation of aggregates. For intermediate $\mathrm{pH}$ values a mixture of Sc-RDC and ro-RDC is present, as reflected in the intermediate values of fluorescence emission intensities (Fig. 6). The intensity at particular pH is therefore proportional to the effective concentration of the "active" RDC, i.e. the ro-RDC, hence labeled as $c_{\text {ro }}$. According to Eq. (4) it holds

$$
c_{\mathrm{ro}}(\mathrm{pH})=c S(\mathrm{pH}) .
$$

At low enough $\mathrm{pH}$, for example at $\mathrm{pH}=3.5$, more or less all the probe will be in the ringopen form, so it means that the total concentration of the probe $c$ will correspond to $c_{\text {ro }}$. 


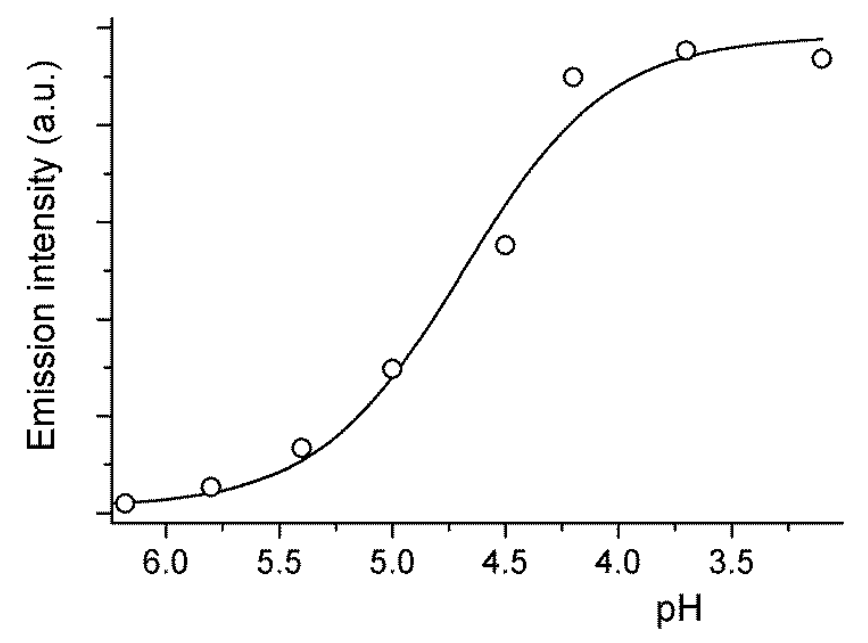

Fig. 6. Dependence of the fluorescence emission intensity of RDC $(c=0.1 \mu \mathrm{M}) \mathrm{on} \mathrm{pH}$. Points show experimental data. The line represents a fitting curve according to Eq. (4).

Due to the non-planar shape of Sc-RDC (see Fig. 5A) the dimerization between scRDC molecules themselves does not seem to be likely. The possibility of dimerization between Sc-RDC and ro-RDC cannot be excluded, although it could be expected to be less probable than the ro-RDC/ro-RDC dimerization. Even if Sc-RDC/ro-RDC dimerization is possible, the energy transfer between these two structural forms is not. So, the observed effect on $\Delta \lambda_{\max }$ (Fig. 4) originates in the formation of ro-RDC/ro-RDC dimers, i.e. $\Delta \lambda_{\max }$ depends on the effective concentration $c_{\text {ro }}$, and $K_{\mathrm{D}}$ does not depend on $\mathrm{pH}$.

It is clear from discussion above that $c_{\text {ro }}$ depends on $\mathrm{pH}$, as indicated in Eq. (6). In addition, from experimental points in Fig. 7A, it can be also seen that the scaling factor $A$ that indicates the extent of the spectral shift, see Eq. (5), is smaller at higher $\mathrm{pH}$. This means that a relative number of ro-RDC/ro-RDC dimers at particular concentration decreases at higher $\mathrm{pH}$, which might be an indication of the presence of sc-RDC/ro-RDC interaction. This interaction causes less available ro-RDC for the ro-RDC/ro-RDC dimerization. The amount of non-fluorescent sc-RDC drops with $\mathrm{pH}$ according to $S(\mathrm{pH})$ (Fig. 6), and with that also the probability for the sc-RDC/ro-RDC interaction. Consequently, the value of the scaling factor $A$ (obtained for $\mathrm{pH}=3.5$ ) will be less affected at lower $\mathrm{pH}$. By making the most straightforward assumption $A(\mathrm{pH})=A S(\mathrm{pH})$ and considering Eqs. (5) and (6) we finally get

$$
\Delta \lambda_{\max }[c, \mathrm{pH}]=A(\mathrm{pH}) x_{\mathrm{D}}\left[c_{\mathrm{ro}}(\mathrm{pH}), K_{\mathrm{D}}\right]=A S(\mathrm{pH}) x_{\mathrm{D}}\left[c S(\mathrm{pH}), K_{\mathrm{D}}\right] .
$$



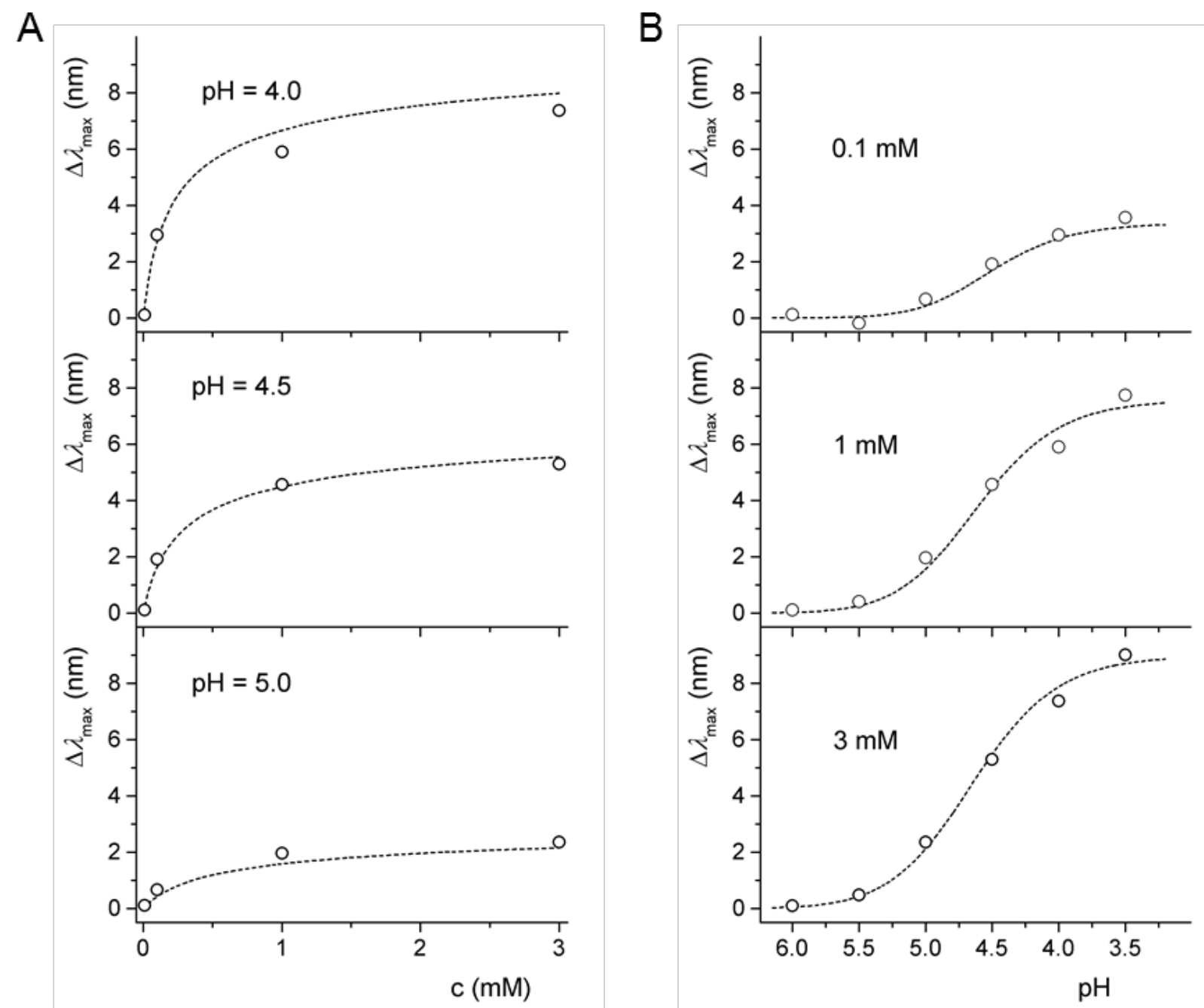

Fig. 7. Dependence of the fluorescence emission spectral maximum shift $\Delta \lambda_{\max }(A)$ on the concentration of RDC at particular $\mathrm{pH}$, and (B) on $\mathrm{pH}$ at particular concentrations of RDC. Each point represents an average value from two different experiments. Estimate of the uncertainty in the $\Delta \lambda_{\max }$ shift is $\pm 0.3 \mathrm{~nm}$. The dashed lines represent calculated (and not fitted) curves according to the model stated in Eq. (7). The parameters used in the model were obtained from fits as explained in the text.

This model was used to draw the lines in Fig. 7 at particular pH (Fig. 7A) and for particular total concentrations $c$ (Fig. 7B) indicated in the figure. The logarithmic nature of $\mathrm{pH}$ causes slow increase at first and then a quick rise in the spectral shift with $\mathrm{pH}$ in Fig. 7B. After the midpoint RDC molecules are approaching the total conversion to the ring-open form so the dimer concentration and consequently the shift level off. It can be seen that the proposed model nicely corresponds to the experimental data presented in Fig. 7, in support of the above reasoning. It is important to note that the values of all parameters used in this model were taken from the fits presented above, i.e. $A$ and $K_{D}$ from the fit of $\Delta \lambda_{\max }(c)$ (Fig. 4), 
and $k$ and $\mathrm{pK}_{\mathrm{a}}$ from the fitted $S(\mathrm{pH})$ curve (Fig. 6). To emphasize the fact that the curves in Fig. 7 are obtained from the model and not by fitting, dashed lines are used instead of solid lines in Fig. 7.

A possible influence of ionic strength should be taken into account for $\mathrm{pH}$ dependence measurements with RDC, given that the ionic strength changes with $\mathrm{pH}$ for the citrate/phosphate buffer. By lowering pH from 6.0 to 3.5 it decreases from about 0.34 $\mathrm{M}$ to about $0.12 \mathrm{M}$ [38], which could in principle lead to somewhat weaker aggregation [5] and gradually smaller $\Delta \lambda_{\max }$ values than anticipated. Consequently, the value of the scaling factor $A$ in Eq. (7), reflecting the magnitude of the spectral maximum shift, could be underestimated. But this does not contradict the observation that aggregation is stronger at lower $\mathrm{pH}$, on the contrary, it supports it. Moreover, the change in ionic strength from $0.05 \mathrm{M}$ to $0.2 \mathrm{M}$ did not cause any significant spectral shift in case of another rhodaminebased probe [18]. Hence, it is not expected that this variable would have a noteworthy influence on our results.

The explained $\mathrm{pH}$ activation can be exploited to detect RDC localization in environment with low $\mathrm{pH}$. Such activatable probes can be very useful for intracellular $\mathrm{pH}$ monitoring probes $[39,40]$. They are especially advantageous for application in spatially resolved (microspectroscopy) experiments in heterogeneous environments, for example in cellular environment, where local concentrations are not known. Namely, differences in spectral properties, as opposed to changes in intensity, cannot be mistakenly attributed to differences in local concentration. At particular $\mathrm{pH}$ the spectral shift directly reflects the local probe concentration at molecular level (see Fig. 7A). Therefore, the spectral shift can be exploited as an indicator of molecular vicinity through the contact-based interaction.

To demonstrate a possible application of spatially confined aggregation-induced effects, we recently presented a fluorescence microspectroscopy (FMS) study of internalization of RDC by dendritic cells (DCs) [24]. The strong increase of fluorescence after internalization is mainly concentrated in small dot-like structures, consistent with $\mathrm{pH}$ activation in endosomes/lysosomes. Since an FMS image contains information of the fluorescence emission spectrum in each image point, experiments were able to reveal spectral red-shift in pixels, where high-enough local concentration induces aggregation. Since this shift is only a few $\mathrm{nm}$ (see Fig. 7) a highly spectrally sensitive FMS technique was used [22,41]. The observed aggregation-induced emission spectral shift shows that accumulation of RDC occurs in low-pH cellular structures. This offers additional understanding of the mechanism of internalization in DCs. 
It has to be noted that beside $\mathrm{pH}$ also presence of different metal ions or macromolecules could affect the aggregation process. For example, metal ions can trigger structural change of particular rhodamine-based probes from spirolactam (spirocyclic) to ring-open [42]. In endosomes/lysosomes $\mathrm{Fe}^{3+}, \mathrm{Fe}^{2+}, \mathrm{Zn}^{2+}$, and $\mathrm{Cu}^{2+}$ can be present [43], and as shown recently an R6G-based probe could be activated by $\mathrm{Fe}^{3+}$ [44]. But even if the observed spectral change in dendritic cells was a consequence of the combined effect of $\mathrm{pH}$, metal ions and macromolecules, the aggregation and the subsequent spectral shift can only be a consequence of the local accumulation of RDC. Thus, the qualitative conclusions from our imaging experiments on dendritic cells would remain the same.

\section{CONCLUSIONS}

Although it is often assumed that non-fluorescent $\mathrm{H}$-dimers prevail in aqueous solutions of rhodamines, both fluorescence excitation and emission spectra reveal the presence of intermediate and deviated geometries from ideal $\mathrm{H}$-dimers. The main aggregation effect observed in the emission spectra is the red-shift. We showed that for R6G in the studied concentration range the shift of the emission spectral maximum is proportional to the dimer mole fraction. Similar behavior was observed for synthesized R6G-based pH-activatable fluorescent probe RDC. The dependence of the shift was analyzed with respect to the probe concentration and to the value of $\mathrm{pH}$. It was shown that the magnitude of the shift at particular $\mathrm{pH}$ directly reports about the probe concentration. Usefulness of such smart probes was demonstrated in a recent experiment. Measurement of the spatial dependence of the aggregation-induced emission spectral shift by fluorescence microspectroscopy has confirmed that RDC targets and accumulates in dendritic cell compartments with low $\mathrm{pH}$. The newly synthesized probe may therefore serve as a model compound for other receptor-specific and environment-sensitive smart probes exploiting the mechanism of aggregation-induced emission spectral shift. 
This work was financially supported by the Slovenian Research Agency (research program No. P1-0060), by the EU H2020 project SmartNanoTox under grant agreement No. 686098 , and in the framework of the Center of Excellence NAMASTE. We are grateful to Prof. Igor Križaj from Jožef Stefan Institute for access to the Tecan microplate reader. The authors acknowledge Prof. Janez Mravljak for providing a part of fluorescence emission data.

\section{REFERENCES}

[1] M. Ogawa, N. Kosaka, P.L. Choyke, H. Kobayashi, H-type dimer formation of fluorophores: a mechanism for activatable, in vivo optical molecular imaging, ACS Chem. Biol. 4 (2009) 535-546. doi:10.1021/cb900089j.

[2] H. Kobayashi, P.L. Choyke, Target-cancer-cell-specific activatable fluorescence imaging probes: rational design and in vivo applications, Acc. Chem. Res. 44 (2011) 83-90. doi:10.1021/ar1000633.

[3] J. Sloniec, U. Resch-Genger, A. Hennig, Photophysics and release kinetics of enzymeactivatable optical probes based on $\mathrm{H}$-dimerized fluorophores on self-immolative linkers, $\mathrm{J}$. Phys. Chem. B. 117 (2013) 14336-14344. doi:10.1021/jp409388b.

[4] A.S. Klymchenko, Solvatochromic and fluorogenic dyes as environment-sensitive probes: Design and biological applications, Acc. Chem. Res. 50 (2017) 366-375. doi:10.1021/acs.accounts.6b00517.

[5] O. Valdes-Aguilera, D.C. Neckers, Aggregation phenomena in xanthene dyes, Acc. Chem. Res. 22 (1989) 171-177. doi:10.1021/ar00161a002.

[6] T.P. Burghardt, J.E. Lyke, K. Ajtai, Fluorescence emission and anisotropy from rhodamine dimers, Biophys. Chem. 59 (1996) 119-131. doi:10.1016/0301-4622(95)00118-2.

[7] P.-Y. Huang, J.-Y. Gao, C.-Y. Song, J.-L. Hong, Ionic complex of a rhodamine dye with aggregation-induced emission properties, Faraday Discuss. 196 (2017) 177-190. doi:10.1039/C6FD00146G.

[8] M. Kasha, H. Rawls, M. El-Bayoumi, The exciton model in molecular spectroscopy, Pure Appl. Chem. 11 (1965) 371-392. doi:10.1351/pac196511030371.

[9] L. Antonov, G. Gergov, V. Petrov, M. Kubista, J. Nygren, UV-Vis spectroscopic and chemometric study on the aggregation of ionic dyes in water, Talanta. 49 (1999) 99-106. doi:10.1016/S0039-9140(98)00348-8.

[10] H. Wang, Q. Yang, L. Sun, S. Wang, W. Wang, C. Zhang, Y. Li, S. Xu, Y. Li, Aggregation states of rhodamine 6G in electrospun nanofibrous films, J. Colloid Interface Sci. 341 (2010) 224-231. doi:10.1016/j.jcis.2009.09.044. 
[11] R. Khodarahmi, R. Yazdanparast, Suppression effect of guanidine hydrochloride on acyclodextrin-assisted refolding of denatured $\alpha$-amylase, Process Biochem. 40 (2005) 29732979. doi:10.1016/j.procbio.2005.01.018.

[12] P. Bojarski, A. Matczuk, C. Bojarski, A. Kawski, B. Kukliński, G. Zurkowska, H. Diehl, Fluorescent dimers of rhodamine $6 \mathrm{G}$ in concentrated ethylene glycol solution, Chem. Phys. 210 (1996) 485-499. doi:10.1016/0301-0104(96)00141-3.

[13] F.L. Arbeloa, I.L. Gonzalez, P.R. Ojeda, I.L. Arbeloa, Aggregate formation of rhodamine 6G in aqueous solution, J. Chem. Soc. Faraday Trans. 2. 78 (1982) 989. doi:10.1039/f29827800989.

[14] L. Malfatti, T. Kidchob, D. Aiello, R. Aiello, F. Testa, P. Innocenzi, Aggregation states of rhodamine 6G in mesostructured silica films, J. Phys. Chem. C. 112 (2008) 16225-16230. doi:10.1021/jp801392f.

[15] K. Kemnitz, K. Yoshihara, Entropy-driven dimerization of xanthene dyes in nonpolar solution and temperature-dependent fluorescence decay of dimers, J. Phys. Chem. 95 (1991) 60956104. doi:10.1021/j100169a012.

[16] H. Tajalli, A. Ghanadzadeh Gilani, M.S. Zakerhamidi, M. Moghadam, Effects of surfactants on the molecular aggregation of rhodamine dyes in aqueous solutions, Spectrochim. Acta Part A Mol. Biomol. Spectrosc. 72 (2009) 697-702. doi:10.1016/j.saa.2008.09.033.

[17] S. Terdale, A. Tantray, Spectroscopic study of the dimerization of rhodamine 6G in water and different organic solvents, J. Mol. Liq. 225 (2017) 662-671.

doi:10.1016/j.molliq.2016.10.090.

[18] J. Humpolíčková, K. Procházka, M. Hof, Octadecylrhodamine B as a specific micelle-binding fluorescent tag for fluorescence correlation spectroscopy studies of amphiphilic watersoluble block copolymer micelles. Spectroscopic behavior in aqueous media, Collect. Czechoslov. Chem. Commun. 68 (2003) 2105-2119. doi:10.1135/cccc20032105.

[19] F. del Monte, J.D. Mackenzie, D. Levy, Rhodamine fluorescent dimers adsorbed on the porous surface of silica gels, Langmuir. 16 (2000) 7377-7382. doi:10.1021/la000540+.

[20] V. Martínez Martínez, F. López Arbeloa, J. Bañuelos Prieto, I. López Arbeloa, Characterization of rhodamine $6 \mathrm{G}$ aggregates intercalated in solid thin films of laponite clay. 2 Fluorescence spectroscopy, J. Phys. Chem. B. 109 (2005) 7443-7450. doi:10.1021/jp050440i.

[21] F.M. Zehentbauer, C. Moretto, R. Stephen, T. Thevar, J.R. Gilchrist, D. Pokrajac, K.L. Richard, J. Kiefer, Fluorescence spectroscopy of Rhodamine 6G: Concentration and solvent effects, Spectrochim. Acta Part A Mol. Biomol. Spectrosc. 121 (2014) 147-151. doi:10.1016/j.saa.2013.10.062.

[22] Z. Arsov, I. Urbančič, M. Garvas, D. Biglino, A. Ljubetič, T. Koklič, J. Štrancar, Fluorescence microspectroscopy as a tool to study mechanism of nanoparticles delivery into living cancer cells, Biomed. Opt. Express. 2 (2011) 2083-2095. doi:10.1364/BOE.2.002083.

[23] E. Sezgin, F. Schneider, V. Zilles, I. Urbančič, E. Garcia, D. Waithe, A.S. Klymchenko, C. Eggeling, Polarity-Sensitive Probes for Superresolution Stimulated Emission Depletion Microscopy, Biophys. J. (2017) 1-10. doi:10.1016/j.bpj.2017.06.050.

[24] Z. Arsov, U. Švajger, J. Mravljak, S. Pajk, A. Kotar, I. Urbančič, J. Štrancar, M. Anderluh, Internalization and accumulation in dendritic cells of a small $\mathrm{pH}$-activatable glycomimetic fluorescent probe as revealed by spectral detection, ChemBioChem. 16 (2015) 2660-2667. doi:10.1002/cbic.201500376.

[25] C.A. Parker, W.T. Rees, Fluorescence spectrometry. A review, Analyst. 87 (1962) 83-111. doi:10.1039/an9628700083.

[26] F. del Monte, D. Levy, Formation of fluorescent rhodamine B J-dimers in sol-gel glasses induced by the adsorption geometry on the silica surface, J. Phys. Chem. B. 102 (1998) 8036-8041. doi:10.1021/jp982396v. 
[27] F.L. Arbeloa, P.R. Ojeda, I.L. Arbeloa, Dimerization and trimerization of rhodamine 6G in aqueous solution. Effect on the fluorescence quantum yield, J. Chem. Soc. Faraday Trans. 2. 84 (1988) 1903. doi:10.1039/f29888401903.

[28] N. Kuzkova, O. Popenko, A. Yakunov, Application of Temperature-Dependent Fluorescent Dyes to the Measurement of Millimeter Wave Absorption in Water Applied to Biomedical Experiments, Int. J. Biomed. Imaging. 2014 (2014) 1-5. doi:10.1155/2014/243564.

[29] S.A. Viznyuk, P.P. Pashinin, A.M. Prokhorov, S.F. Rastopov, A.T. Sukhodolskii, Temperature-induced luminescence rise in aqueous solutions of rhodamine 6G, JETP Lett. 47 (1988) 230-233.

[30] A. Penzkofer, W. Leupacher, Fluorescence behaviour of highly concentrated rhodamine 6G solutions, J. Lumin. 37 (1987) 61-72. doi:10.1016/0022-2313(87)90167-0.

[31] K.A. Selanger, J. Falnes, T. Sikkeland, Fluorescence lifetime studies of Rhodamine $6 \mathrm{G}$ in methanol, J. Phys. Chem. 81 (1977) 1960-1963. doi:10.1021/j100535a016.

[32] R.F. Chen, J.R. Knutson, Mechanism of fluorescence concentration quenching of carboxyfluorescein in liposomes: Energy transfer to nonfluorescent dimers, Anal. Biochem. 172 (1988) 61-77. doi:10.1016/0003-2697(88)90412-5.

[33] P. Ilich, P.K. Mishra, S. Macura, T.P. Burghardt, Direct observation of rhodamine dimer structures in water, Spectrochim. Acta Part A Mol. Biomol. Spectrosc. 52 (1996) 1323-1330. doi:10.1016/0584-8539(96)01719-9.

[34] M. Hida, T. Sanuki, Studies of the aggregation of dyes. The scope of application of the maximum-slope method, Bull. Chem. Soc. Jpn. 43 (1970) 2291-2296.

doi:10.1246/bcsj.43.2291.

[35] M.M. Wong, Z.A. Schelly, Solvent-jump relaxation kinetics of the association of rhodamine type laser dyes, J. Phys. Chem. 78 (1974) 1891-1895. doi:10.1021/j100612a002.

[36] K. Ajtai, T.P. Burghardt, Conformation of xanthene dyes in the sulfhydryl 1 binding site of myosin, Biochemistry. 34 (1995) 15943-15952. doi:10.1021/bi00049a009.

[37] W. Zhang, B. Tang, X. Liu, Y. Liu, K. Xu, J. Ma, L. Tong, G. Yang, A highly sensitive acidic $\mathrm{pH}$ fluorescent probe and its application to HepG2 cells, Analyst. 134 (2009) 367-371. doi:10.1039/b807581f.

[38] P.J. Elving, J.M. Markowitz, I. Rosenthal, Preparation of buffer systems of constant ionic strength, Anal. Chem. 28 (1956) 1179-1180. doi:10.1021/ac60115a034.

[39] J. Han, K. Burgess, Fluorescent indicators for intracellular pH, Chem. Rev. 110 (2010) 2709-2728. doi:10.1021/cr900249z.

[40] R. Wang, C. Yu, F. Yu, L. Chen, C. Yu, Molecular fluorescent probes for monitoring pH changes in living cells, TrAC Trends Anal. Chem. 29 (2010) 1004-1013. doi:10.1016/j.trac.2010.05.005.

[41] I. Urbančič, Z. Arsov, A. Ljubetič, D. Biglino, J. Štrancar, Bleaching-corrected fluorescence microspectroscopy with nanometer peak position resolution, Opt. Express. 21 (2013) 25291-25306. doi:10.1364/OE.21.025291.

[42] H.N. Kim, M.H. Lee, H.J. Kim, J.S. Kim, J. Yoon, A new trend in rhodamine-based chemosensors: application of spirolactam ring-opening to sensing ions, Chem. Soc. Rev. 37 (2008) 1465. doi:10.1039/b802497a.

[43] H. Xu, D. Ren, Lysosomal Physiology, Annu. Rev. Physiol. 77 (2015) 57-80. doi:10.1146/annurev-physiol-021014-071649.

[44] Y. Liu, R. Shen, J. Ru, X. Yao, Y. Yang, H. Liu, X. Tang, D. Bai, G. Zhang, W. Liu, A reversible rhodamine $6 \mathrm{G}$-based fluorescence turn-on probe for $\mathrm{Fe} 3+$ in water and its application in living cell imaging, RSC Adv. 6 (2016) 111754-111759.

doi:10.1039/C5RA09758D. 